\title{
Space Charge and AC Electric Breakdown Strength in Polyethylene
}

\author{
Churui Zhou, George Chen \\ The Tony Davies High Voltage Laboratory \\ University of Southampton \\ SouthamptonSO17 1BJ, United Kingdom \\ cz3g13@soton.ac.uk
}

\begin{abstract}
The effect of space charge on dielectric performances is generally neglected under high voltage alternate current (HVAC) electric fields, due to the limited charge amount accumulated. This paper intends to expound the characteristics of space charge in polymeric under AC stresses and its importance to the breakdown strength of polyethylene. A numerical model based on space charge dynamics under high voltage electric fields has been used to explain the differences of the breakdown strength in polyethylene under AC and DC stresses. Bipolar charge transport theory is adopted to analyse the charge transportation processes. Space charge profiles at the time of breakdown occurrence under different electric fields (including DC voltage, $0.5,5$ and $50 \mathrm{~Hz} \mathrm{AC}$ voltages) and in the samples with different thicknesses (from 50 to $200 \mu \mathrm{m}$ ) are simulated. The relationship between space charge and the breakdown strength is discussed. The simulated results agree well with the trend of experimental breakdown results: generally, breakdown occurs easier under AC stress than DC stress; the breakdown strength is increased with the decrease of the sample thickness. The results suggest the presence of space charge under AC stresses has a great impact on electric breakdown strength of the material.
\end{abstract}

Keywords-AC electric breakdown; space charge; Bipolar charge transport; Low density polyethylene (LDPE)

\section{INTRODUCTION}

The breakdown strength is an essential electrical characteristic of an insulation material, and the electric breakdown and ageing failure of insulating are two of the major problems related to the reliability of power systems. Several different mechanisms, including electronic, avalanche, electromechanical, thermal breakdowns, and partial discharges have been proposed to explain the origin of the phenomenon. Recently, some works reveal the strongly relationship between the breakdown strength and the space charge dynamics within insulation [1,2]. In 2012, Chen et al applied a bipolar charge transport model to simulate LDPE's electric breakdown strength under dc conditions [3]. Their results demonstrated the breakdown strength of LDPE is strongly related to the thickness of testing sample and the ramping rate of the testing voltage. An inversed power's law trend describing the breakdown strength versus testing sample thickness was obtained, which is in agreement with the existing theory proposed by Kim [4]. And they also demonstrated and emphasized the influence of the external voltage ramping rate on the dc breakdown strength. In [3], Chen et al used simulation parameters setting in [5], where the movement of the holes dominates the overall charge transportation process. The amount of trapped positive charges is obviously larger than negative charges. Under this circumstance, severe electric field distortion will happen when a large amount of positive charges and negative charges meet. It has been found that the charge dynamics, such as the encounter of negative and positive charges, are affected greatly by the thickness of the sample. A reduction in breakdown strength versus the thickness has been obtained when the thickness of the sample increases. However their simulation was focused on breakdown strength under HVDC conditions, and charge dynamics are expected to be significantly different under HVAC stresses. Trapped charges within LDPE under HVAC will be much less, and currently no evidence supports that the positive charges will dominate the overall charge movements under HVAC. In this paper, a general simulation model for both AC and DC conditions has been proposed to study the space charge dependant breakdown process. The computing time step for the simulation is $0.4 \mathrm{~ms}$, and the spatial coordinate step is $0.1 \mu \mathrm{m}$. The parameter selection for the simulation is tuned more towards matching the measured space charge profiles under HVAC in [6]. The proposed model is more suitable for the general analysis of the breakdown process under AC, DC and the superimposed fields to understand the charge dynamics behind the electric breakdown.

\section{NUMERICAL SimULATION MODEL}

\section{A. Bipolar Charge Transport Model}

Charge transportation in dielectrics can be described using three basic equations. The total flux $j(x, t)$ is regarded as a time and space dependent variable. Its combination with Poisson equation and continuity equation can be used to analyse the behaviour of charge carriers in the system. The overall process neglects the effects of charge carrier's diffusion.

$$
\begin{gathered}
\frac{\partial E(x, t)}{\partial x}=\frac{\rho(x, t)}{\varepsilon} \\
j(x, t) \stackrel{=}{=} \mu n(x, t) E(x, t) \\
\frac{\partial n(x, t)}{\partial t}+\frac{\partial j(x, t)}{\partial x}=s
\end{gathered}
$$

where $\mu$ is the mobility of carriers, $\mathrm{n}$ is the density of mobile species, $E$ is the electric field, $j$ is the current density, $x$ is the spatial coordinate, $t$ is the time, $s$ is the source term, $\varepsilon$ is the dielectric permittivity, and $\rho$ is the net charge density. 
In the bipolar charge model, charge carriers can be injected from both electrodes, according to the polarity of the external fields. The charge injection process is analysed using a Schottky mechanism and charge injection is considered to be the only source of charge carriers. After charges penetrating into the material, the applied field will influence their movements across the material, and an effective mobility is proposed to study this process. During charge carriers movement, some will be trapped in the localised states (deep trap centres) and therefore, the total amount of mobile charges across the sample will be reduced. However, no extraction barrier is introduced in the model, in order to simplify the computing procedure. Besides, positive and negative charges are prone to recombine with each other (electrons with holes).

\section{B. Numerical model setting and results analysis}

In this work, the parameters are chosen according the comparison of the simulated charge profiles with the measured space charge profiles under HVAC. The results from the measured charge dynamics in [6] (as shown in Fig.1) are applied for the breakdown simulation model. Charge profiles were obtained from the PEA measurements from samples(LDPE) directly after AC ageing with various times. The aged samples were transferred to the PEA system within $5 \mathrm{~min}$ and the space charge formed during the ageing was examined without the externally applied dc field.

The results clearly show that the negative charges accumulated in the bulk of the sample. It was explained based on the higher mobility of the injected electrons. Therefore the parameters are chosen accordingly. Moreover, the parameter of the maximum trapped density in the simulation model may be not very important as the amount of both of the negative and positive charges is generally very small under AC stresses. And the final parameter setting for breakdown strength is 500 $\mathrm{kV} / \mathrm{mm}$ as presented in Table I [5, 7].

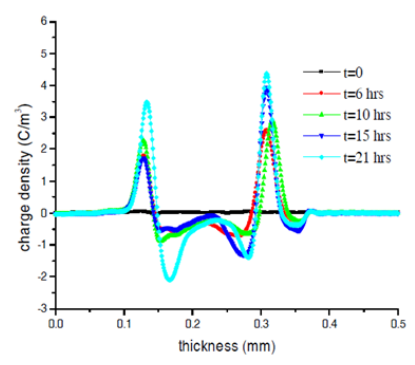

(a)

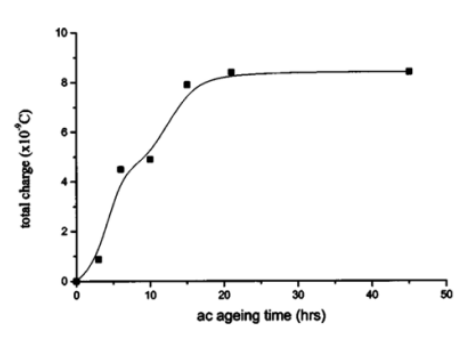

(b)
Fig. 1 Charge distribution in ac aged LDPE (a) and total charge amount vs ageing time (b) with ageing stress of $50 \mathrm{kV} / \mathrm{mm}$ [6]

The simulated charge profiles under a constant $50 \mathrm{kV} / \mathrm{mm}$ HVAC stress are presented in Fig. 2, and the simulated charge density profiles are similar to the measured results shown in Fig.1 (a). It can be observed that some positive charges distribute in the narrow regions adjacent to the electrodes in the simulation. However, these positive charges are difficult to be distinguished from the induced charges at electrodes in the experiment due to the limited resolution of the PEA system. Besides from the calculated electric fields results, it is clear that the maximum electric field distortion locates in the vicinities of the electrodes. And it is also obvious that the overall charge distribution curves are dominated by the trapped holes and electrons, as mobile charges are not able to move further into the bulk region under AC electric stresses.

Table I Parameters for charge dynamics simulation of breakdown strength test

\begin{tabular}{|c|c|c|}
\hline Parameter & Value & Unit \\
\hline \multicolumn{3}{|l|}{ Barrier height for injection } \\
\hline $\mathrm{W}_{\mathrm{ei}}$ (electrons) & 1.1 & $\mathrm{eV}$ \\
\hline $\mathrm{W}_{\mathrm{hi}}$ (holes) & 1.12 & $\mathrm{eV}$ \\
\hline Low field Mobility $\mu_{\mathrm{E} 0}$ & $9 \times 10^{-16}$ & $\mathrm{~m}^{2} \mathrm{~V}^{-1} \mathrm{~s}^{-1}$ \\
\hline Low field Mobility $\mu_{\mathrm{h} 0}$ & $3 \times 10^{-16}$ & \\
\hline Power law's index of mobility $n$ & 1.165 & $\mathrm{~m}^{2} \mathrm{~V}^{-1} \mathrm{~s}^{-1}$ \\
\hline \multicolumn{3}{|l|}{ Trap density } \\
\hline $\mathrm{N}_{0 \mathrm{et}}$ (electrons) & 100 & $\mathrm{Cm}^{-3}$ \\
\hline $\mathrm{N}_{\text {Oht }}$ (holes) & 100 & $\mathrm{Cm}^{-3}$ \\
\hline \multicolumn{3}{|l|}{ Trapping coefficients } \\
\hline Be (electrons) & $7 \times 10^{-3}$ & $\mathrm{~s}^{-1}$ \\
\hline Bh (holes) & $7 \times 10^{-5}$ & $\mathrm{~s}^{-1}$ \\
\hline \multicolumn{3}{|l|}{ Recombination coefficients } \\
\hline $\mathrm{S}_{0}$ trapped electron-trapped hole & $4 \times 10^{-3}$ & $\mathrm{~m}^{3} \mathrm{C}^{-1} \mathrm{~s}^{-1}$ \\
\hline $\mathrm{S}_{1}$ mobile electron-trapped hole & $4 \times 10^{-3}$ & $\mathrm{~m}^{3} \mathrm{C}^{-1} \mathrm{~s}^{-1}$ \\
\hline $\mathrm{S}_{2}$ trapped electron-mobile hole & $4 \times 10^{-3}$ & $\mathrm{~m}^{3} \mathrm{C}^{-1} \mathrm{~s}^{-1}$ \\
\hline $\mathrm{S}_{3}$ mobile electron-mobile hole & 0 & $\mathrm{~m}^{3} \mathrm{C}^{-1} \mathrm{~s}^{-1}$ \\
\hline Temperature $\mathrm{T}$ & 300 & $\mathrm{~K}$ \\
\hline Intrinsic breakdown strength & $5 \times 10^{8}$ & $\mathrm{Vm}^{-1}$ \\
\hline
\end{tabular}

In real breakdown tests, the voltage is applied from two electrodes to the sample with a chosen ramping rate until the breakdown occurs. A ramp breakdown tester normally can test samples up to $0.1 \mathrm{~mm}$ thick, and the range of ramp rates varies from 10 to $300 \mathrm{~V} / \mathrm{s}$. a typical ramp rate for samples under DC stresses is $100 \mathrm{~V} / \mathrm{s}$, whilst $50 \mathrm{~V} / \mathrm{s}$ for AC stresses [8, 9]. Therefore in the numerical modelling the external applied voltage is defined as following:

$$
\mathrm{V}=\mathrm{Rt} \sin (2 \pi \mathrm{ft})
$$

where $\mathrm{R}$ is the ramping rate and $\mathrm{f}$ is the external voltage frequency. In the simulation process, $\mathrm{R}$ and $\mathrm{f}$ can be changed according to the analysis requirements.
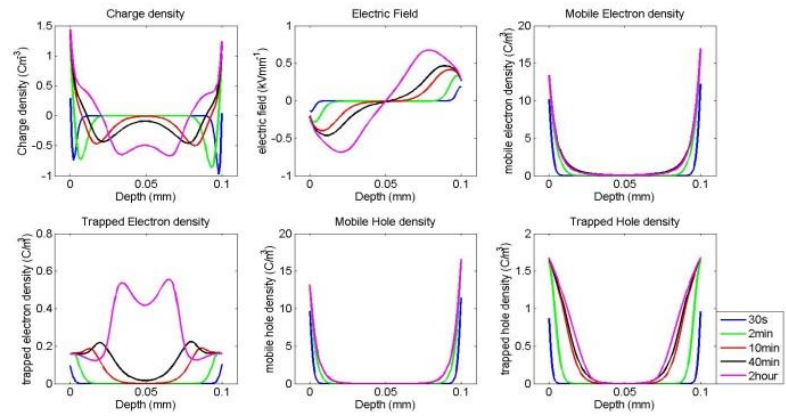

Fig. 2 Simulated charge profiles under 50kV/mm HVAC stress at phase 0 using breakdown strength simulation model

The simulated material is the low density polyethylene with a thickness ranging from 50 to $200 \mu \mathrm{m}$. In the existing experimental results from the literature [2,3], it is generally believed that the breakdown strength for polyethylene is around $500 \mathrm{kV} / \mathrm{mm}$. Thus in the simulation, when the internal local field reaches $500 \mathrm{kV} / \mathrm{mm}$, the simulation stops, and the applied electric field (which is regarded as the apparent breakdown strength) is obtained from the current applied voltage divided by the sample thickness. Simulated charge distribution and electric field results at the cycle of breakdown occurrence are illustrated in Fig. 3 and Fig. 4. The simulation condition is a $50 \mu \mathrm{m}$ LDPE under $50 \mathrm{~Hz}$ HVAC stress within a ramping rate of $50 \mathrm{~V} / \mathrm{s}$, and the breakdown strength for this 
condition is $160.8 \mathrm{kV} / \mathrm{mm}$. This computed result is close to the measured results obtained by Hussin et al [8]. In [8], following the analysis using Weibull distribution on the breakdown data of 50um LDPE under $50 \mathrm{~Hz} \mathrm{AC}$ with a ramping rate of $50 \mathrm{~V} / \mathrm{s}$, the characteristic breakdown strength of $192.2 \mathrm{kV} / \mathrm{mm}$ was obtained, with a $90 \%$ lower and uppers bounds of $148 \mathrm{kV} / \mathrm{mm}$ and $226 \mathrm{kV} / \mathrm{mm}$ respectively.

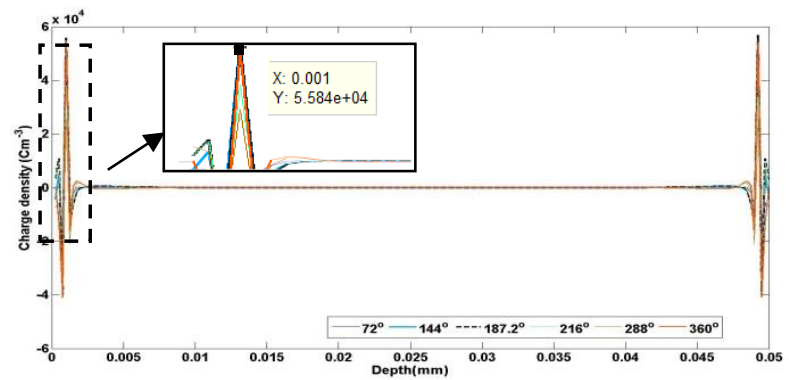

Fig. 3 Charge profiles corresponding to breakdown cycle for 50um LDPE under $50 \mathrm{~Hz} \mathrm{HVAC}$ with a ramping rate of $50 \mathrm{~V} / \mathrm{s}$

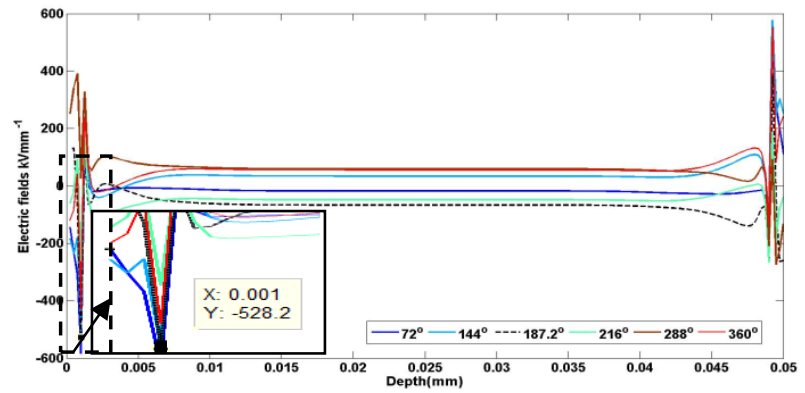

Fig. 4 Electric fields profiles corresponding to breakdown cycle for 50um LDPE under $50 \mathrm{~Hz} \mathrm{HVAC}$ with a ramping rate of $50 \mathrm{~V} / \mathrm{s}$
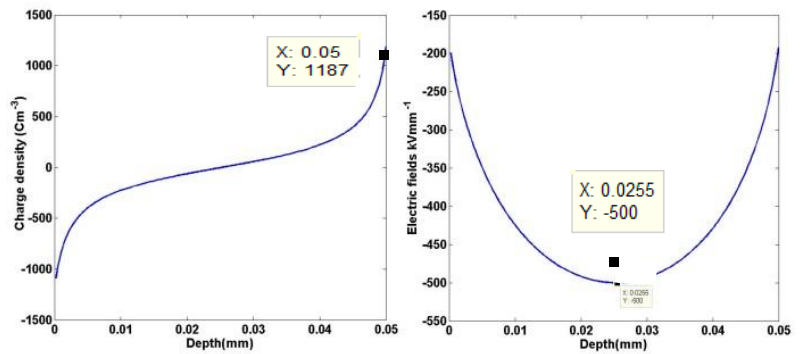

Fig. 5 Charge and electric fields profiles at the breakdown for 50um LDPE under HVDC with a ramping rate of 50V/s

Fig. 5 presents the simulated profiles of DC charge and fields at breakdown time in LDPE using the same simulating parameter setting as for AC. Comparing with the AC results, the DC breakdown results show a much higher breakdown strength of $421.8 \mathrm{kV} / \mathrm{mm}$ and a different location of breakdown. Under AC conditions, the highest fields' distortion normally happens near the electrodes while for DC situation, it generally occurs in the central bulk of the testing sample. Although the integral area charge density at breakdown time is smaller under AC stress $\left(6.93 \times 10^{-3} \mathrm{C} / \mathrm{m}^{2}\right)$ than DC stress $\left(1.24 \times 10^{-2} \mathrm{C} / \mathrm{m}^{2}\right)$, the maximum peak value of the charge density is significantly larger under AC stress $\left(5.58 \times 10^{4} \mathrm{C} / \mathrm{m}^{3}\right)$ than DC stress $\left(1.19 \times 10^{3} \mathrm{C} / \mathrm{m}^{3}\right)$. This adjacent electrode charge peak obtained under ac condition distorts the local electric field severely (twice larger than the applied fields). Therefore, the local fields reaching the breakdown strength more quickly under the ramping $\mathrm{AC}$ stress as the space charges accumulate within a narrow region near the electrodes and distort the local electric fields greatly. On the other hand, the charge under DC conditions can transport further into the bulk region of the sample, and the maximum electric fields distortion happens in the centre where the holes and electrons encounter each other. Therefore the local fields under ramping DC voltage will be more difficult to reach the breakdown strength, comparing with the AC voltage.

\section{THE RELATIONSHIP OF BREAKDOWN STRENGTHS AND TESTING SAMPLE THICKNESS}

The relationship between the solid dielectrics and their breakdown strengths has been sufficiently studied over the years and it is generally believed an inverse power law is reasonable to analyse the relationship [4].

$$
\mathrm{E}(\mathrm{d})=\mathrm{kd}^{-\mathrm{n}}
$$

where $\mathrm{E}$ is the applied electric field at breakdown and $\mathrm{k}$ and $\mathrm{n}$ are two constants that are associated with the testing material.

In this section, the relationship of the sample thickness and breakdown strengths is analysed and discussed using the simulated results with a range of thickness from $50 \mu \mathrm{m}$ to $200 \mu \mathrm{m}$ with a fixed voltage ramping rate of $100 \mathrm{~V} / \mathrm{s}$. And the influences of the external voltage frequency on the breakdown strengths and strengths' relationship with sample thickness are also demonstrated. Fig. 6 presents the simulated breakdown strength under different frequencies, together with a computed result for dc conditions using the same setting. Then a power's law trend fitting is applied to the data in order to clearly demonstrate the breakdown strengths' relationship with sample thickness.

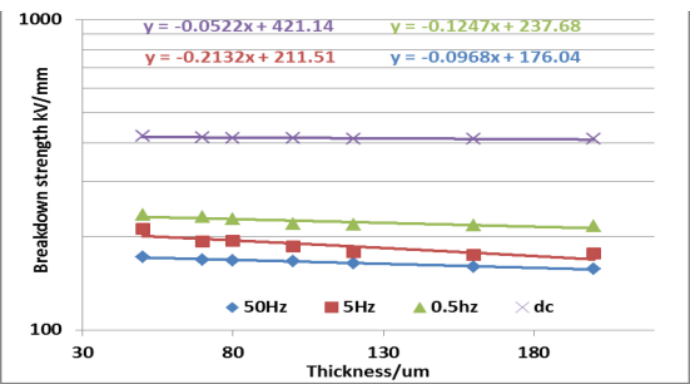

Fig.6 Breakdown strengths under different frequencies along within testing sample thickness increasing

It is clearly from Fig. 6 that the simulated breakdown strength under HVAC decreases along with frequency increasing. And the breakdown strength under DC conditions can be regarded as an extreme case (external voltage frequency is zero), which has a higher breakdown strength than those under AC. The simulated breakdown strength relationship with sample thickness is similar to those in literatures [2, 4, 9]. And using these simulated results, the significant role of space charge in electric breakdown processes has been proved. The distorted local electric field due to charge formation within the insulation is the key reason leading to breakdown of the insulation. And for thicker samples, the localised charges will become more difficult to transport to the other side.

The strength decreasing trends along with frequency enhancing are consistent with the measured breakdown strength of LDPE [9], where breakdown strengths of 25um LDPE from $60 \mathrm{~Hz}$ to $1000 \mathrm{~Hz}$ and $0 \mathrm{~Hz}$ under $25^{\circ} \mathrm{C}$ and $70^{\circ} \mathrm{C}$ 
were measured. The results are a little higher than those presented in [8] and the simulated results. The difference may be due to the different sample thicknesses and measurement environments.

The reason for this phenomenon is similar to the reason for a lower breakdown strength under AC comparing with DC conditions. Because of the constant changing polarity of the outer external fields, charges accumulated near to the electrodes under the $\mathrm{AC}$ electric field are much more than those trapped under DC conditions. $\left(5.58 \times 10^{4} \mathrm{C} / \mathrm{m}^{3}\right.$ for $\mathrm{AC}$ and $1.19 \times 10^{3} \mathrm{C} / \mathrm{m}^{3}$ for DC in Fig.3 and Fig.5) When the frequency of the AC fields increases, the increasing amount of charges accumulates adjacent to the electrodes. Due to the more frequent alternation of the external fields' polarity, it becomes difficult for the charges moving further into the bulk of the insulating materials. Specific breakdown locations of 50um LDPE under various frequencies with a ramping rate of $50 \mathrm{~V} / \mathrm{s}$ are presented in Table II. It is obviously that the location of the breakdown is moving towards the electrodes along with frequency increasing. The breakdown location is more close to the electrodes, only a smaller charge amount is needed to make the local fields reaching the breakdown strength. Therefore, the apparent breakdown strengths should be decreasing along with frequency.

Table II Breakdown locations vs. the frequency of external fields for 50umLDPE under ramping rate of 50V/s

\begin{tabular}{|l|l|l|l|}
\hline External voltage frequency & $0.5 \mathrm{~Hz}$ & $5 \mathrm{~Hz}$ & $50 \mathrm{~Hz}$ \\
\hline $\begin{array}{l}\text { Location from the electrodes } \\
\text { where breakdown is initiated }\end{array}$ & $4.5 \mu \mathrm{m}$ & $1.25 \mu \mathrm{m}$ & $0.75 \mu \mathrm{m}$ \\
\hline
\end{tabular}

\section{IV.THE RELATIONSHIP OF BREAKDOWN STRENGTH AND EXTERNAL VOLTAGE RAMPING RATE}

It has been reported that the ramping rates of the applied electric fields can influence the measured breakdown strength (the apparent breakdown strength) of the material. Different ramping rates have been used to measure the breakdown strength over the years [4, 8-9]. In order to compare the apparent breakdown strength obtained by the measurements with different ramping rates, the effect of the ramping rate on LDPE breakdown strength is analysed and discussed using the bipolar charge transport model.

Fig 7 presents the computed breakdown strength under HVAC and HVDC. For both conditions, the material breakdown strength increases along with the ramping rate. Rapid increase happens from $50 \mathrm{~V} / \mathrm{s}$ to $250 \mathrm{~V} / \mathrm{s}$. Similar trend has been observed [10]. However the ramping rate seems to have a more obvious influence on breakdown strength under AC conditions. From 50V/s to 500V/s, a change of more than $100 \mathrm{kV} / \mathrm{mm}$ has been observed. This gives a good explanation to the remarkable differences of testing breakdown strength for materials under ac conditions within the literature regardless of the different testing equipment along with diverse testing environments. In this way, the issue of external voltage ramping rate should be paid more attention when considering/comparing breakdown strength under $\mathrm{AC}$ electric fields.

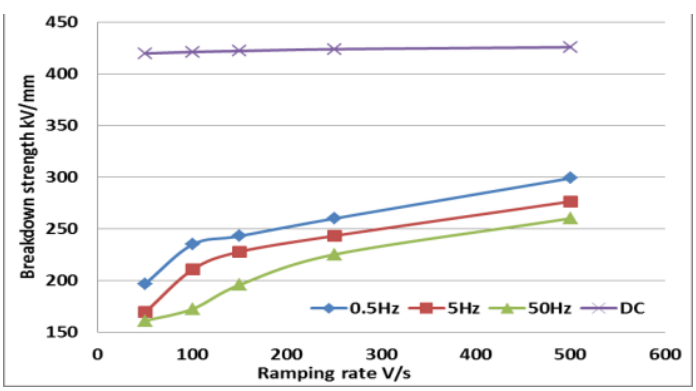

Fig. 7 Breakdown strengths under different outer fields' ramping rate and frequencies

\section{CONCLUSION AND FUTURE WORK}

In this paper a bipolar charge transport model has been used to analyse the breakdown strengths' relationship with sample thickness, external voltage ramping rate and voltage frequency. A power's law with negative exponential index can be utilised to predict the breakdown strength of different thicknesses of the insulating material. The results demonstrate that the distortion of electric fields caused by the charge dynamics is the essential reason leading to the electric breakdown of insulation. Moreover, the lower AC apparent breakdown strength, compared with DC one, results from the significantly distorted field in the vicinities of the electrodes that can quickly reach to the breakdown strength of the LDPE sample under ramping voltage. In future, experiments will be done to validate the simulated results and revise the numerical model presented if necessary.

\section{REFERENCES}

[1] A. Bradwell, R. Cooper, and B. Varlow, "Conduction in polythene with strong electric fields and the effect of prestressing on the electric strength," in Proceedings of the Institution of Electrical Engineers, 1971, pp. $247-254$

[2] K. Matsui, Y. Tanaka, T. Takada, T. Fukao, K. Fukunaga, T. Maeno, et al., "Space charge behavior in low density polyethylene at prebreakdown," Dielectrics and Electrical Insulation, IEEE Transactions on, vol. 12, pp. 406-415, 2005.

[3] G. Chen, J. Zhao, S. Li, and L. Zhong, "Origin of thickness dependent dc electrical breakdown in dielectrics," Applied Physics Letters, vol. 100, p. 222904, 2012.

[4] H. Kim and F. Shi, "Thickness dependent dielectric strength of a lowpermittivity dielectric film," Dielectrics and Electrical Insulation, IEEE Transactions on, vol. 8, pp. 248-252, 2001.

[5] G. Chen and J. W. Zhao, "Observation of negative differential mobility and charge packet in polyethylene," Journal of Physics D-Applied Physics, vol. 44, Jun 12011.

[6] G. Chen, M. Fu, X. Z. Liu, and L. S. Zhong, "ac aging and space-charge characteristics in low-density polyethylene polymeric insulation," Journal of Applied Physics, vol. 97, Apr 152005.

[7] J. Zhao, Z. Xu, G. Chen, and P. L. Lewin, "Numeric description of space charge in polyethylene under ac electric fields," Journal of Applied Physics, vol. 108, Dec 152010.

[8] N. Hussin, J. Zhao, and G. Chen, "The AC breakdown and Space Charge Characteristics of LDPE in the presence of Crosslinking Byproduct," in Electrical Insulating Materials (ISEIM), Proceedings of 2011 International Conference on, 2011, pp. 65-68.

[9] M. Nagao, K. Takano, Y. Mizuno, and M. Kosaki, "Intrinsic Ac Breakdown of Low-Density Polyethylene Film above RoomTemperature," Proceedings of the 3rd International Conference on Properties and Applications of Dielectric Materials, Vols 1 and 2, pp. 1165-1168,1991.

[10] C. Chauvet and C. Laurent, "Weibull statistics in short-term dielectric breakdown of thin polyethylene films," Electrical Insulation, IEEE Transactions on, vol. 28, pp. 18-29, 1993. 